OPEN ACCESS

Edited by:

Mikhail Lebedev,

Duke University, United States

Reviewed by:

Marco lacoboni,

University of California, Los Angeles,

United States

Vassiliy Tsytsarev,

University of Maryland, College Park, United States

Mayank A. Jog,

Keck School of Medicine of USC,

United States

*Correspondence:

Águida S. Foerster

foerster.as@gmail.com; foerster@ifado.de

Anirban Dutta

anirband@buffalo.edu;

adutta@case.edu

Specialty section:

This article was submitted to

Neural Technology,

a section of the journal

Frontiers in Neuroscience

Received: 27 October 2017

Accepted: 12 June 2018

Published: 04 July 2018

Citation:

Foerster ÁS, Rezaee Z, Paulus W, Nitsche MA and Dutta A (2018)

Effects of Cathode Location and the Size of Anode on Anodal Transcranial Direct Current Stimulation

Over the Leg Motor Area in Healthy

Humans. Front. Neurosci. 12:443. doi: 10.3389/fnins.2018.00443

\section{Effects of Cathode Location and the Size of Anode on Anodal Transcranial Direct Current Stimulation Over the Leg Motor Area in Healthy Humans}

\author{
Águida S. Foerster ${ }^{1,2 *}$, Zeynab Rezaee ${ }^{3}$, Walter Paulus ${ }^{1}$, Michael A. Nitsche ${ }^{2,4}$ and \\ Anirban Dutta ${ }^{3 *}$ \\ ${ }^{1}$ Department of Clinical Neurophysiology, Universitätsmedizin Göttingen, Georg-August-Universität Göttingen, Göttingen, \\ Germany, ${ }^{2}$ IfADo - Leibniz Research Center for Working Environment and Human Factors, Dortmund, Germany, \\ ${ }^{3}$ Department of Biomedical Engineering, University at Buffalo, The State University of New York (SUNY), Buffalo, NY, \\ United States, ${ }^{4}$ Department of Neurology, BG University Hospital Bergmannsheil, Bochum, Germany
}

Objective: Non-invasive brain stimulation such as transcranial direct current stimulation (tDCS) involves passing low currents through the brain and is a promising tool for the modulation of cortical excitability. In this study, we investigated the effects of cathode location and the size of anode for anodal tDCS of the right-leg area of the motor cortex, which is challenging due to its depth and orientation in the inter-hemispheric fissure.

Methods: We first computationally investigated the effects of cathode location and the size of the anode to find the best montage for specificity of stimulation effects for the targeted leg motor area using finite element analysis (FEA). We then compared the best electrode montage found from FEA with the conventional montage (contralateral supraorbital cathode) via neurophysiological testing of both, the targeted as well as the contralateral leg motor area.

Results: The conventional anodal tDCS electrode montage for leg motor cortex stimulation using a large-anode $(5 \mathrm{~cm} \times 7 \mathrm{~cm}$, current strength $2 \mathrm{~mA})$ affected the contralateral side more strongly in both the FEA and the neurophysiological testing when compared to other electrode montages. A small-anode $(3.5 \mathrm{~cm} \times 1 \mathrm{~cm}$ at $0.2 \mathrm{~mA})$ with the same current density at the electrode surface and identical contralateral supraorbital cathode placement improved specificity. The best cathode location for the small-anode in terms of specificity for anodal tDCS of the right-leg motor area was T7 (10-10 EEG system).

Conclusion: A small-anode $(3.5 \mathrm{~cm} \times 1 \mathrm{~cm})$ with the same current density at the electrode surface as a large-anode $(5 \mathrm{~cm} \times 7 \mathrm{~cm})$ resulted in similar cortical excitability alterations of the targeted leg motor cortex respresentation. In relation to the other stimulation conditions, the small-anode montage with the cathode positioned at T7 resulted in the best specificity.

Keywords: lower limb motor cortex, stimulation parameters, motor cortex excitability, modeling, transcranial direct current stimulation (tDCS) 


\section{INTRODUCTION}

Clinical applications of non-invasive brain stimulation (NIBS) are currently an evolving area and increasingly used as an adjuvant treatment during motor rehabilitation (Flöel, 2014). Transcranial direct current stimulation (tDCS) is a NIBS modality that involves application of low intensity direct currents using two or more electrodes for a certain duration, which can alter corticospinal excitability polarity-dependently for up to $60 \mathrm{~min}$ after the end of the stimulation (Bailey et al., 2016). The first studies were conducted in the hand area of the motor cortex that showed corticospinal excitability alterations, monitored by transcranial magnetic stimulation (TMS)-induced motor evoked potentials (MEP) (Rossi et al., 2009), of up to 40\%. In the motor cortex, excitability enhancement was achieved by anodal stimulation, whereas cathodal stimulation reduced excitability (Nitsche and Paulus, 2000). Moreover, the strength and duration of these after-effects are controlled by current intensity and duration (Nitsche and Paulus, 2001; Nitsche et al., 2003b; MonteSilva et al., 2010, 2013; Batsikadze et al., 2013). Pharmacological studies (Liebetanz et al., 2002; Nitsche et al., 2003a) identified a role of tDCS-induced membrane polarization and NMDA receptor activation for these sustained after-effects (Nitsche and Paulus, 2001).

Awareness about the relevance of computational modeling for rational design of electrode montages, taking into account not only the electric field strength but also the current flow direction in relation to neuronal orientation (Das et al., 2016), has increased recently. Computational modeling can help to identify optimal electrode positions, and improve efficacy of stimulation (Datta et al., 2011). In this study, we focused on the application of tDCS over the leg area of the motor cortex, which presents a challenge due to its depth and orientation in the interhemispheric fissure, and has not been explored as much as tDCS of the hand area of the motor cortex. Some studies, however, have shown that tDCS can modulate the excitability of the leg area of the motor cortex. Jeffery et al. (2007) showed that $10 \mathrm{~min}$ of stimulation with the anode over the leg area of the motor cortex in healthy humans increased corticospinal excitability of the anterior tibial (TA) muscle by up to $59 \%$ compared to baseline values for up to $60 \mathrm{~min}$ after stimulation. Cathodal tDCS, however, did not decrease corticospinal excitability. In a functional outcome study in healthy humans, anodal tDCS has been shown to transiently enhance maximal leg pinch force for up to $30 \mathrm{~min}$ after stimulation compared to baseline, but did not affect reaction time (Tanaka et al., 2009). Also here, cathodal tDCS did not alter performance. Roche et al. (2011) showed that anodal tDCS over the same area induced effects on spinal network excitability similar to those observed during co-contraction of lower-limb muscles. Such indirect effects on spinal network excitability may be suited to support postural stability and balance, as shown by the recent studies conducted in healthy humans (Dutta et al., 2014a; Kaminski et al., 2016).

Regarding clinical application of tDCS over the primary motor cortex leg area, anodal stimulation on the lesioned cortex with a large square sponge electrode $(5 \mathrm{~cm} \times 5 \mathrm{~cm})$ with $2 \mathrm{~mA}$ for $10 \mathrm{~min}$ improved balance and strengthened the affected lower limb in stroke patients (Sohn et al., 2013). Jayaram and Stinear (2009) explored the effects of anodal tDCS over the lesioned motor cortex of nine chronic stroke survivors using a small $8.1 \mathrm{~cm}^{2}$ saline-soaked sponge electrode as anode (unlike most other studies, which used relatively large $25-36 \mathrm{~cm}^{2}$ stimulation electrodes) whose edge was aligned to the midsagittal plane, and a large $36 \mathrm{~cm}^{2}$ cathode which was placed above the contralateral orbit. They investigated bilateral modulatory effects of stimulation on the tibialis anterior (TA), medial gastrocnemius, medial hamstrings, and vastus lateralis muscles. Anodal tDCS over the ipsilesional motor cortex increased paretic limb and decreased non-paretic limb motor excitability, and thus showed a relatively focal effect. Regarding effects on motor functions, a single session of anodal tDCS of the paretic lower limb was shown to increase knee extensor force for up to $30 \mathrm{~min}$ following stimulation in hemiparetic stroke survivors (Tanaka et al., 2011). van Asseldonk and Boonstra (2016) showed similar montage-related performance differences in 10 healthy subjects and 10 chronic stroke survivors that also revealed a large inter-individual variability of effects. In that study, two montages with a $5 \mathrm{~cm} \times 7 \mathrm{~cm}$ anode placed over the lesioned hemisphere with the short edge of the rectangular electrode aligned to the mid-sagittal fissure and centered over the motor cortex representation of the leg, and the cathode placed over the supraorbital region (called unihemispheric montage) or over the motor cortex contralateral to the targeted area (called bihemispheric montage) were compared. In the study of van Asseldonk and Boonstra (2016), subjects with the largest effect for one montage often showed opposite effects for the other. This underscores the relevance of the placement of the electrodes when aiming to stimulate the leg area, analogous to what has been described for the hand area (Bikson et al., 2010; Moliadze et al., 2010). Placement of the electrodes is not only critical for the electric field strength, but also electric field direction (Rawji et al., 2018). Both factors are relevant for stimulation of the leg area of the motor cortex due to its depth and orientation in the inter-hemispheric fissure. However, a comprehensive finite element modeling of tDCS of the leg motor area with a realistic head model and physiological validation of the computational results has not been conducted so far. Stimulation parameters and brain anatomy affect efficacy and specificity of $\mathrm{tDCS}$, which is particularly challenging for cortical areas not on the brain surface such as the leg area of the motor cortex.

In our preliminary study (Dutta et al., 2012) using a simple three-shell head model, we hypothesized that not only the electric field strength but also the electric field direction is relevant for the effects of anodal tDCS over the leg motor area. For the present study, our goal was to maximally stimulate the targeted leg motor representation while avoiding stimulation of the contralateral leg motor volume. We investigated simple two-electrode unihemispheric montages using a realistic computational head model and explored the impact of cathode placement and anode size on anodal tDCS over the motor cortex leg area. We then evaluated the appropriateness of the computational models via neurophysiological testing in healthy individuals. 


\section{MATERIALS AND METHODS}

\section{Finite Element Model of the Human Head}

The head model for finite element modeling was developed using the freely available SimNIBS software pipeline. ${ }^{1}$ The SimNIBS software pipeline (Windhoff et al., 2013) uses fat-suppressed T1-weighted magnetic resonance images (MRI) as input for FreeSurfer (Fischl, 2012). We used the Colin27 average brain (Collins et al., 1998; Holmes et al., 1998), which is the stereotaxic average of 27 T1-weighted MRI scans of the same individual, to create the head model (see iso2mesh toolbox (iso2mesh; Fang and Boas, 2009). The Colin27 average brain has been widely adopted as a stereotaxic template that includes and labels cerebellum, brain stem, and ventricles. After segmentation, different components like scalp, skull, cerebrospinal fluid (CSF), white matter (WM), and gray matter (GM) of the brain were modeled as different volume conductors with their own specific conductivity (Windhoff et al., 2013), as shown in Table 1.

The anode and cathode injected a specified amount of current (source) in the volume conductor. The electrodes were modeled as saline-soaked sponge cuboids (see section "Electrode Montages for Finite Element Modeling"). We analyzed the headmodel for electric field distribution using the Finite Element Method (FEM), provided in the SimNIBS pipeline, which provides a powerful numerical tool to solve the required partial differential equations (PDE).

The quasi-static formula for direct current stimulation is given below,

$$
-\nabla \cdot(\sigma \nabla V)=S \text { in } \Omega
$$

where $\Omega$ is the volume conductor, $V_{(x, y, z)}$ is the scalar potential field, $\sigma_{(x, y, z)}$ is the conductivity tensor, $S$ is the source term. The Dirichlet boundary condition is presented in Section "Electrode Montages for Finite Element Modeling". FEM divides the volume conductor into spatial elements and nodes for discrete computations of the PDE. The tetrahedral head meshes for FEM were generated using the "mri2mesh" tool in the SimNIBS software pipeline (Windhoff et al., 2013) with an average tetrahedron volume of $1 \mathrm{~mm}^{3}$. The continuity of the solution is maintained at the boundary of the elements using shape function objects. The electric field values at the nodes within the bilateral leg area cluster in the cortical tissue (not CSF) were captured by Boolean intersection with a sphere of $1 \mathrm{~cm}$ radius centered at $(-7 \mathrm{~mm},-38 \mathrm{~mm}, 75 \mathrm{~mm})$ and $(6 \mathrm{~mm},-38 \mathrm{~mm}, 75 \mathrm{~mm})$ in the MNI coordinates, as shown in Figure 1. The cortical tissue

${ }^{1}$ www.simnibs.org

TABLE 1 | Electrical conductivity.

\begin{tabular}{lc}
\hline Component & Electrical conductivity $\left(\mathbf{S ~}^{\mathbf{- 1}}\right)$ \\
\hline Scalp & 0.465 \\
Skull & 0.010 \\
CSF & 1.654 \\
Gray matter & 0.276 \\
White matter & 0.126
\end{tabular}

cluster after Boolean intersection with the sphere was comparable to the functional MRI activation volume $\left(\sim 1450 \mathrm{~mm}^{3}\right)$ during plantar $\left(45^{\circ}\right)$ and dorsal flexion $\left(10^{\circ}\right)$ of the foot at a rate of approximately $0.5 \mathrm{~Hz}$ (Alkadhi et al., 2002). All node values within the cortical tissue clusters were imported in Matlab (The Mathworks, Inc., United States) to compute the average magnitude and direction (described in section "Optimization of Electrode Montage").

\section{Electrode Montages for Finite Element Modeling}

The electrode positions were defined with fiducials at Nz, Iz, right, and left preauricular points for registration with the head model in accordance with the 10-10 system defined in Oostenveld and Praamstra (2001). We explored the effects of the following electrode positions, and sizes: motor cortex anode (large: $5 \mathrm{~cm} \times 7 \mathrm{~cm}$ at $2 \mathrm{~mA}$ and small: $3.5 \mathrm{~cm} \times 1 \mathrm{~cm}$ at $0.2 \mathrm{~mA}$ ) at the approximate TA muscle hotspot based on neurophysiological testing (Dutta et al., 2014b) - $15 \mathrm{~mm}$ left lateral and $20 \mathrm{~mm}$ posterior to $\mathrm{Cz}(1 \mathrm{~mm},-28 \mathrm{~mm}, 87 \mathrm{~mm})$. The cathode $(5 \mathrm{~cm} \times 7 \mathrm{~cm})$ was placed at Fp1 $(-21 \mathrm{~mm}, 70 \mathrm{~mm}, 15 \mathrm{~mm})$, F7 (-53 mm, $32 \mathrm{~mm}, 2 \mathrm{~mm})$, T7 $(-70 \mathrm{~mm},-16 \mathrm{~mm},-8 \mathrm{~mm})$, P7 $(-58 \mathrm{~mm},-65 \mathrm{~mm},-6 \mathrm{~mm}), \mathrm{Oz}(1 \mathrm{~mm},-101 \mathrm{~mm}, 6 \mathrm{~mm})$, P8(56 mm, $-64 \mathrm{~mm},-6 \mathrm{~mm}), \mathrm{T} 8(55 \mathrm{~mm}, 30 \mathrm{~mm},-1 \mathrm{~mm})$, and Fp2 $(25 \mathrm{~mm}, 68 \mathrm{~mm}, 15 \mathrm{~mm})$. Here, $(x, y$, and $z)$ refer to the MNI stereotaxic space (Jurcak et al., 2007); the $x$ direction is mediolateral, the $y$ direction anterior-posterior, and the $z$ direction ventro-dorsal. This resulted in eight montages with cortical projection of their respective electrode center denoted using the 10-10 EEG system (Koessler et al., 2009). The contralateral supraorbital cathode position (Fp2) was termed "conventional montage," since this montage was most often used in prior tDCS studies of the leg motor area (Madhavan and Shah, 2012). These eight electrode montages were evaluated computationally, as described in Section "Optimization of Electrode Montage," based on the Colin27 FEM head model (see section "Finite Element Model of the Human Head"). Transcranially injected direct current per unit area at the top of the saline-soaked sponge anode was set constant at $0.057 \mathrm{~mA} / \mathrm{cm}^{2}$ which was a Dirichlet boundary condition for the FEM head model.

\section{Optimization of Electrode Montage}

The tDCS current per unit area at the top of the sponge electrodes was kept constant at $0.057 \mathrm{~mA} / \mathrm{cm}^{2}$ for the computational optimization of the electrode montage that resulted in $2 \mathrm{~mA}$ direct current for the large-anode and $0.2 \mathrm{~mA}$ for the smallanode. This current amplitude is considered to be safe and adequate for experimental validation in healthy humans (Nitsche et al., 2003b). The electric field values (see section "Finite Element Model of the Human Head") at the nodes within the bilateral leg motor volume were extracted with the "CutSphere" command of $\mathrm{Gmsh}^{2}$ (Geuzaine and Remacle, 2009) and imported in Matlab (The Mathworks Inc., United States) as a text file for computing their average magnitude and direction. Here, the

${ }^{2}$ http://gmsh.info/ 


\section{A Targeted motor cortex sphere centered at $(-7 \mathrm{~mm},-38 \mathrm{~mm}, 75 \mathrm{~mm})$ in the MNI coordinates}
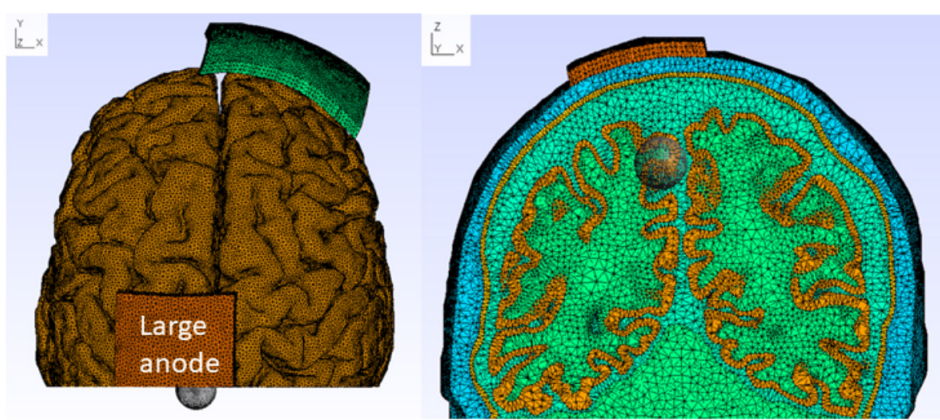

B Contralateral motor cortex sphere centered at $(6 \mathrm{~mm},-38 \mathrm{~mm}, 75 \mathrm{~mm})$ in the MNI coordinates
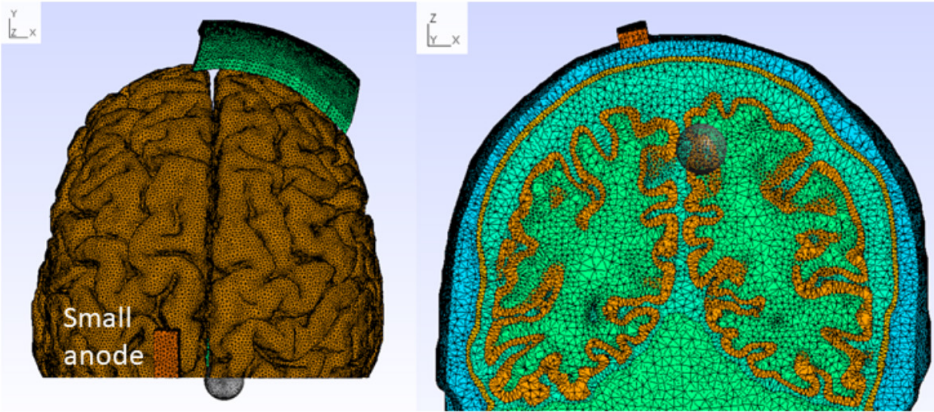

FIGURE 1 | Colin27 FEM head model for the "conventional montage" electrode arrangement with the cathode $(5 \mathrm{~cm} \times 7 \mathrm{~cm})$ at Fp2 (10-10 EEG system) and anode centered at $15 \mathrm{~mm}$ left lateral and $20 \mathrm{~mm}$ posterior to $\mathrm{Cz}$ (10-10 EEG system). (A) Large-anode: $5 \mathrm{~cm} \times 7 \mathrm{~cm}$ at $2 \mathrm{~mA}$, (B) Small-anode: $3.5 \mathrm{~cm} \times 1 \mathrm{~cm}$ at $0.2 \mathrm{~mA}$. The cortical tissue cluster found after Boolean intersection with the sphere of a radius of $1 \mathrm{~cm}$ captures the electric field at the targeted [centered at $(-7 \mathrm{~mm}$,

$-38 \mathrm{~mm}, 75 \mathrm{~mm}$ ) MNI coordinates - A] and the contralateral [centered at (6 mm, $-38 \mathrm{~mm}, 75 \mathrm{~mm}$ ) MNI coordinates - B] leg motor cortex, as shown with top (left panel) and coronal (right panel) sectional views.

cortical tissue cluster found after Boolean intersection of the cortical tissue with the sphere of a radius of $1 \mathrm{~cm}$ with centroids at $(-7 \mathrm{~mm},-38 \mathrm{~mm}, 75 \mathrm{~mm})$ and $(6 \mathrm{~mm},-38 \mathrm{~mm}, 75 \mathrm{~mm})$ in MNI coordinates (see Figure 1) represented the targeted and contralateral leg motor volume respectively. The specificity of the electric field $\underset{E F}{\rightarrow}$ ) for different cathode locations (Fp1, F7, T7, P7, $\mathrm{Oz}, \mathrm{P} 8, \mathrm{~T} 6, \mathrm{~T} 8$, and Fp2) was determined by the laterality of the volume-averaged electric field strength (Opitz et al., 2015) toward the targeted leg motor volume. Therefore, the specificity was computationally (comp) found based on the volume-averaged magnitude of the electric field $(|E F|=\sqrt{E F o E F})$ or volumeaveraged electric field strength (Opitz et al., 2015),

$$
\text { Specificity }_{\text {comp }}^{\text {montage }}=\frac{\left(|E F|_{\text {targeted }}-|E F|_{\text {contralateral }}\right)}{\left(|E F|_{\text {targeted }}+|E F|_{\text {contralateral }}\right)}
$$

The best montage based on the computational (comp) analysis, Specificity comp cone , was compared with the "conventional montage" based on neurophysiological testing (see section "Experimental Validation"). Our goal was to maximally stimulate the targeted leg motor volume [centroid at $(-7 \mathrm{~mm},-38 \mathrm{~mm}$, $75 \mathrm{~mm})$ MNI coordinates] while avoiding stimulation of the contralateral leg motor volume [centroid at $(6 \mathrm{~mm},-38 \mathrm{~mm}$, $75 \mathrm{~mm}$ ) MNI coordinates] - see Figure 1. The volume-averaged electric field $(\underset{E F}{\rightarrow})$ unit vector was also computed for the targeted (targ in Equation 2b) and contralateral (contra in Equation 2b) leg motor volumes, and the angle between these vectors was used for comparison.

$$
\text { Angle }_{\text {comp }}^{\text {montage }}=\angle \overrightarrow{E F}_{\text {targeted }}-\angle \overrightarrow{E F}_{\text {contralateral }}
$$

\section{Experimental Validation}

Twelve healthy subjects, seven males and five females (age: 21-36 years, all right-leg dominant) volunteered for the study. The subjects signed an informed consent form before participation and the study was approved by the Institutional Review Board of the University Medical Center, Goettingen, Germany. The experiment consisted of multiple sessions of anodal or sham tDCS with each session addressing a separate electrode montage (list given in Table 2, complete cross-over design) in randomized order, with sufficient (1 week) "wash-out" time in between the sessions.

The anode was placed over the dominant right-leg motor cortex representation, as shown in Figure 1. Figure 1 also shows the targeted and contralateral leg motor volumes, which were used to compute the specificity of the stimulation. A transcranial DC stimulator (NeuroConn, Germany) delivered the currents for $10 \mathrm{~min}$ via the anode centered on the scalp at the position where TMS of the primary motor cortex elicited maximal twitches in the resting dominant right-leg TA muscle. TMS was delivered with a Magpro Stimulator (MagVenture, United States) through a butterfly coil (MC-B70, MagVenture, 
TABLE 2 | Electrode montages and stimulation parameters for neurophysiological testing.

\begin{tabular}{lcc}
\hline Montage & \multicolumn{1}{c}{ Anode } & Cathode \\
\hline $\begin{array}{l}\text { Large-anode in } \\
\text { conventional montage } \\
\begin{array}{l}\text { Small-anode in } \\
\text { conventional montage }\end{array}\end{array}$ & $5 \mathrm{~cm} \times 7 \mathrm{~cm}$ at $2 \mathrm{~mA}$ & $5 \mathrm{~cm} \times 7 \mathrm{~cm}$ at FP2 \\
$\begin{array}{l}\text { Small-anode in side } \\
\text { montage }\end{array}$ & $3.5 \mathrm{~cm} \times 1 \mathrm{~cm}$ at $0.2 \mathrm{~mA}$ & $5 \mathrm{~cm} \times 7 \mathrm{~cm}$ at FP2 \\
$\begin{array}{l}\text { Small-anode in sham } \\
\text { montage }\end{array}$ & $3.5 \mathrm{~cm} \times 1 \mathrm{~cm}$ at $0 \mathrm{~mA}$ & $5 \mathrm{~cm} \times 7 \mathrm{~cm}$ at T7 \\
\end{tabular}

United States) and the resting muscle activity as well as the MEP were monitored using biofeedback software (Signal 2 software, CED, United Kingdom). For TMS of the right-leg motor area, a right-to-left oriented current flow in the brain tissue is required for MEP generation and conversely, when stimulating the left-leg motor area with TMS, a left-to-right oriented current is optimal. The handle of the TMS butterfly coil was thus aligned approximately $90^{\circ}$ to the parasagittal plane to induce a tissue current that runs in the coronal plane in the required direction (Groppa et al., 2012). The location of the coil on the scalp for the targeted rightleg, called the "target-hotspot," was identified with singlepulse TMS by adjusting the coil position until it resulted in the largest MEP at a moderate suprathreshold stimulation intensity. Then, the contralateral left-leg hotspot, called the "contralateral hotspot," was identified. Both hotspots were marked with water-resistant ink to reduce variability of coil placement during bilateral testing of corticospinal excitability. Corticospinal excitability alterations (Rossini et al., 1999) were evaluated using single-pulse TMS intensity that elicited 10 MEPs of average $0.5 \mathrm{mV}$ amplitude at baseline before intervention. Corticospinal excitability was monitored at the "target-hotspot" as well as the "contralateral-hotspot." Corticospinal excitability was measured before and immediately after the completion of tDCS as well as every $15 \mathrm{~min}$ for the next $60 \mathrm{~min}$, and then every $30 \mathrm{~min}$ for next $60 \mathrm{~min}$ for each session, and $24 \mathrm{~h}$ for the real stimulation conditions, 10 MEPs were recorded for each time bin. For sham tDCS, the current was ramped up for $15 \mathrm{~s}$ and then down to zero in $15 \mathrm{~s}$ for blinding purposes. All subjects included in this study responded at baseline to single-pulse TMS with 10 MEPs of an average $0.5 \mathrm{mV}$ at the "target-hotspot" as well as at the "contralateralhotspot."

During anodal tDCS of the "target-hotspot," the current was ramped up linearly for $15 \mathrm{~s}$ to a constant amplitude of either 2 or $0.2 \mathrm{~mA}$ which was maintained for $10 \mathrm{~min}$ before being ramped down linearly for $15 \mathrm{~s}$.

The specificity of the corticospinal excitability after-effects based on MEP-based neurophysiological (neurophys) measures at the "target-hotspot" and the "contralateral-hotspot" was computed as,

$$
\text { Specificity }_{\text {neurophys }}^{\text {montage }}=\frac{\left(M E P_{\text {targeted }}-M E P_{\text {contralateral }}\right)}{\left(M E P_{\text {targeted }}+M E P_{\text {contralateral }}\right)}
$$

Here, $M E P_{\text {targeted }}$ is the MEP-based measure of corticospinal excitability at the "target-hotspot" and the $M E P_{\text {contralateral is the }}$ one at the "contralateral-hotspot."

Two-way repeated measure ANOVAs (within subject factors: time post-tDCS and tDCS-condition, dependent variables: baseline-normalized MEP and Specificity electrophys $_{\text {mone }}$ ) were conducted to calculate the effect of the tDCS-conditions: large-anode in the "conventional montage," small-anode in the "conventional montage," small-anode in the "side montage," and small-anode in the "sham montage." Pairwise post hoc comparisons were carried out using $t$-statistics with Bonferroni correction ("multcompare" in Matlab). Alpha was set at $P<0.05$.

\section{RESULTS}

The results from the computational modeling of the electric field at the targeted right-leg motor volume [centroid at $(-7 \mathrm{~mm}$, $-38 \mathrm{~mm}, 75 \mathrm{~mm})$ in MNI coordinates] and the contralateral left-leg motor volume [centroid at $(6 \mathrm{~mm},-38 \mathrm{~mm}, 75 \mathrm{~mm})$ in MNI coordinates] are shown in Figure 2. The maximum electric field magnitude at the targeted leg motor volume for the largeanode, $5 \mathrm{~cm} \times 7 \mathrm{~cm}$, at $2 \mathrm{~mA}$, was around $0.4 \mathrm{~V} / \mathrm{m}$, while for the small-anode, $3.5 \mathrm{~cm} \times 1 \mathrm{~cm}$ at $0.2 \mathrm{~mA}$, it was around $0.05 \mathrm{~V} / \mathrm{m}$. Therefore, the maximum electric field strength was about onetenth at the targeted right-leg motor volume with the smallanode (Figures 2B,D) when compared to that for the large-anode (Figures 2A,C). For the small-anode, the maximum electric field strength was found to be higher at the targeted right-leg motor volume than the contralateral left-leg motor volume with the cathode at T7 (Figure 2D) when compared to the cathode at Fp2 (Figure 2B). This difference in the electric field strength was captured with the specificity metric from finite element analysis. The Specificity comp montage $_{\text {for }}$ for large-anode (in black) and small-anode (in gray) for different cathode locations is shown in Figure 3A. The T7 cathode location provided the best specificity for both the large-anode and the small-anode. This best montage identified by computational analysis with the smallanode positioned over the "target-hotspot" and the cathode over T7 was labeled "side montage" for neurophysiological testing. Also, the angle between the average electric field direction (unit vector) at the targeted right-leg and the contralateral left-leg motor volume, Angle comp $_{\text {mone }}$, was compared, and the results are shown in Figure 3B. The small-anode resulted in a larger Angle $\mathrm{e}_{\text {comp }}$ when compared to the large-anode, however the distribution across cathode locations, Fp1, F7, T7, P7, Oz, P8, T6, and Fp2 (10-10 EEG system) was similar for the small-anode and the large-anode.

For neurophysiological evaluation based on Specificity neurophys $_{\text {montage }}=\frac{\left(M E P_{\text {targeted }}-M E P_{\text {contralateral }}\right)}{\left(M E P_{\text {targeted }}+M E P_{\text {contralateral }}\right)}$, the "side montage" was compared with the "conventional montage."

Figure 4 shows the results from the neurophysiological testing of corticospinal excitability changes following anodal tDCS. All results are displayed as mean \pm standard error of means. The corticospinal excitability changes are presented as MEPs individually normalized to baseline (baseline-normalized 

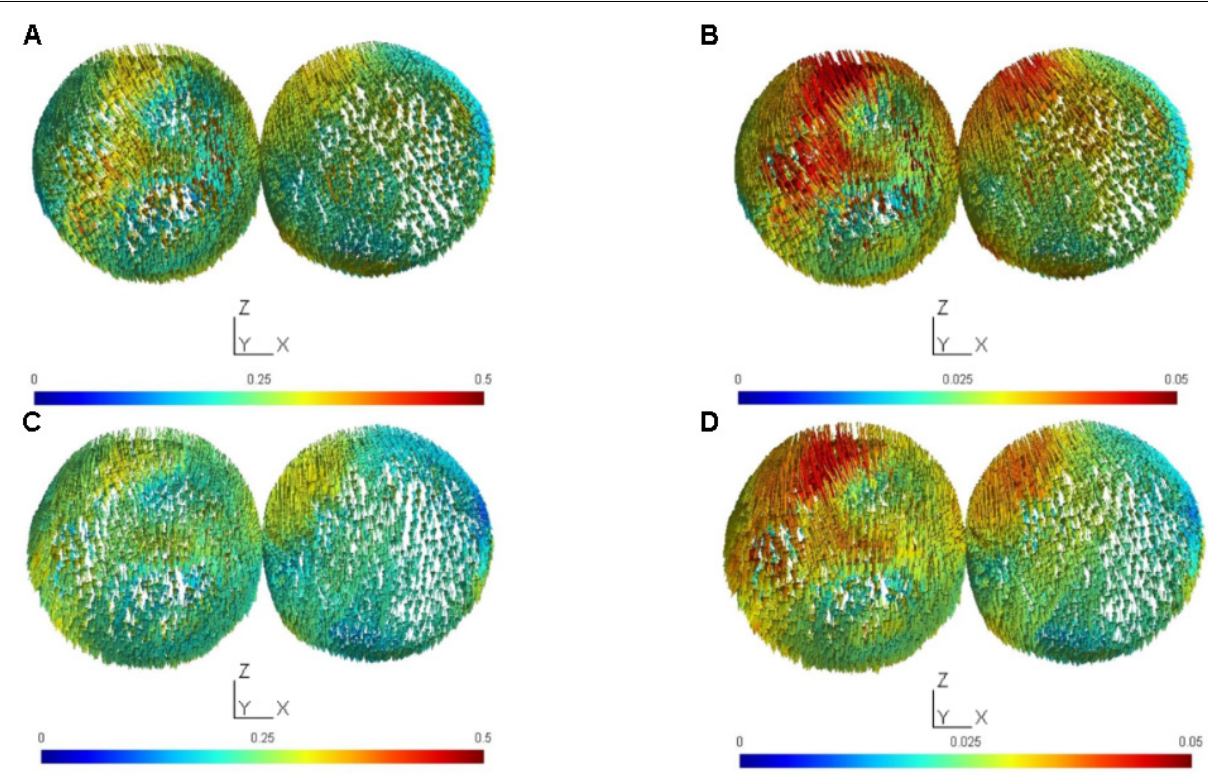

FIGURE 2 | The electric field (EF) vector was computed for the targeted (targ) cortical tissue cluster found after Boolean intersection with the sphere of a radius of $1 \mathrm{~cm}$ centered at $(-7 \mathrm{~mm},-38 \mathrm{~mm}, 75 \mathrm{~mm})$ in MNI coordinates, and the contralateral (contra) sphere with a radius of $1 \mathrm{~cm}$ centered at $(6 \mathrm{~mm},-38 \mathrm{~mm}, 75 \mathrm{~mm})$ in MNI coordinates. (A) target and contralateral clusters for anodal tDCS with the large-anode, $5 \mathrm{~cm} \times 7 \mathrm{~cm}$ at $2 \mathrm{~mA}$ and the cathode placed at Fp2 (10-10 EEG system), (B) target and contralateral clusters for anodal tDCS with the small-anode, $3.5 \mathrm{~cm} \times 1 \mathrm{~cm}$ at $0.2 \mathrm{~mA}$ and the cathode placed at Fp2 (10-10 EEG system), (C) target and contralateral clusters for anodal tDCS with the large-anode, $5 \mathrm{~cm} \times 7 \mathrm{~cm}$ at $2 \mathrm{~mA}$ and the cathode placed at T7 (10-10 EEG system), (D) target and contralateral clusters for anodal tDCS with the small-anode, $3.5 \mathrm{~cm} \times 1 \mathrm{~cm}$ at $0.2 \mathrm{~mA}$ and the cathode placed at T7 (10-10 EEG system). Scales of EF magnitudes are different for the small and large electrodes to make it possible to identify the distribution of field magnitudes for both electrode sizes.

MEP) from the targeted right-leg and the contralateral leftleg TA muscles before and immediately after the completion of anodal tDCS as well as every $15 \mathrm{~min}$ for the next $60 \mathrm{~min}$, and then every $30 \mathrm{~min}$ for the next $60 \mathrm{~min}$ for each session. The repeated measure two-way ANOVA [within subject factors: time post-tDCS(min): $0,15,30,45,60,90,120$, and tDCScondition: large-anode "conventional montage," small-anode "conventional montage," small-anode "side montage," smallanode "sham montage"] conducted for the dependent variable baseline-normalized MEP of the right-leg "target-hotspot" showed significant main effects of time post-tDCS $[F(6)=4.65$, $P<0.05]$ and tDCS-condition $[F(3)=23.44, P<0.05]$, but no significant interaction $[F(18)=1.18, P=0.264]$. For the dependent variable baseline-normalized MEP of the left-leg "contralateral-hotspot," a significant main effect was found only for tDCS-condition $[\mathrm{F}(3)=9.79, \mathrm{P}<0.05]$ but not for time $[F(6)=2.08, P=0.0528]$ or the respective interaction $[F(18)=0.6$, $P=0.9011]$.

The Specificity electrophys $_{\text {moge }}$ of the corticospinal excitability aftereffects for different tDCS conditions is shown in Figure 5 for the single subject level. All results are displayed as mean \pm standard error of means. The post hoc tests using $t$-statistics with Bonferroni correction revealed that the baselinenormalized MEP of the right-leg "target-hotspot" for the small-anode in the "sham montage" was lowest and differed significantly $(P<0.05)$ from the other tDCS-conditions after intervention (Figure 6A). The baseline-normalized MEP of the left-leg "contralateral-hotspot" were highest for the large-anode "conventional montage" and differed significantly $(P<0.05)$ from the other tDCS-conditions (Figure 6B). Consequently, Specificity meurophys $_{\text {nentage }}$ of the corticospinal excitability after-effects, which is the normalized difference between the baselinenormalized MEP of the right-leg "target-hotspot" and the left-leg "contralateral-hotspot" was found to be negative (95\% confidence interval) for the large-anode "conventional montage" in the post hoc tests (see Figure 6C). Post hoc tests revealed that the Specificity montage neurophys was significantly different $(P<0.05)$ for different tDCS-conditions, with the small-anode "side montage" having the highest mean (i.e., best montage based on Specificity neurophys $\left._{\text {nontage }}\right)$, followed by the small-anode "conventional montage," the large-anode "conventional montage," and then the small-anode "sham montage" - see Figure 6C.

\section{DISCUSSION}

The results of this study supply information about the effects of electrode montage and anode size on the specificity of anodal tDCS after-effects on the leg motor area. All active stimulation conditions induced the expected target motor cortex excitability enhancements. Hereby, the small-anode "side montage" configuration, i.e., $3.5 \mathrm{~cm} \times 1 \mathrm{~cm}$ anode placed over the right-leg motor "target-hotspot" with the cathode placed over T7 (10-10 EEG system) was found to be superior to both "conventional montages" with the cathode positioned over Fp2 (10-10 EEG system) in terms of specificity in both the 

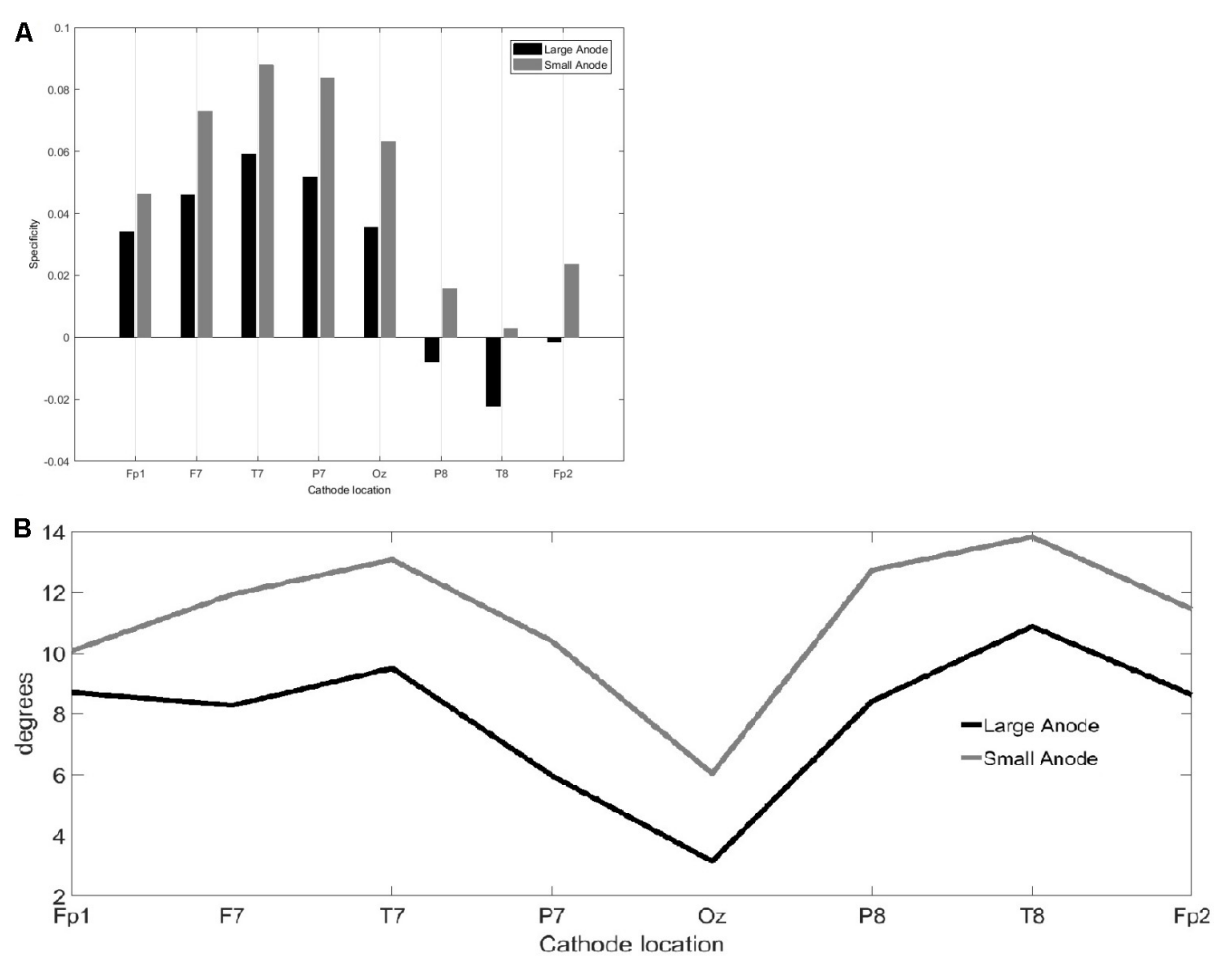

FIGURE 3 | Results from the finite element modeling of the large-anode $(5 \mathrm{~cm} \times 7 \mathrm{~cm}$ at $2 \mathrm{~mA})$ and the small-anode $(3.5 \mathrm{~cm} \times 1 \mathrm{~cm}$ at $0.2 \mathrm{~mA})$ placed at the right-leg "target-hotspot" with different cathode locations (montages), Fp1, F7, T7, P7, Oz, P8, T6, and Fp2 (10-10 EEG system). (A) Bar graph comparing Specificity $y_{\text {comp }}^{\text {mone }}$ from equation 2a. (B) Angle, Angle montage from Equation 2b, of $\overrightarrow{\vec{E} F}$ between the right-leg targeted and the left-leg contralateral motor volumes.
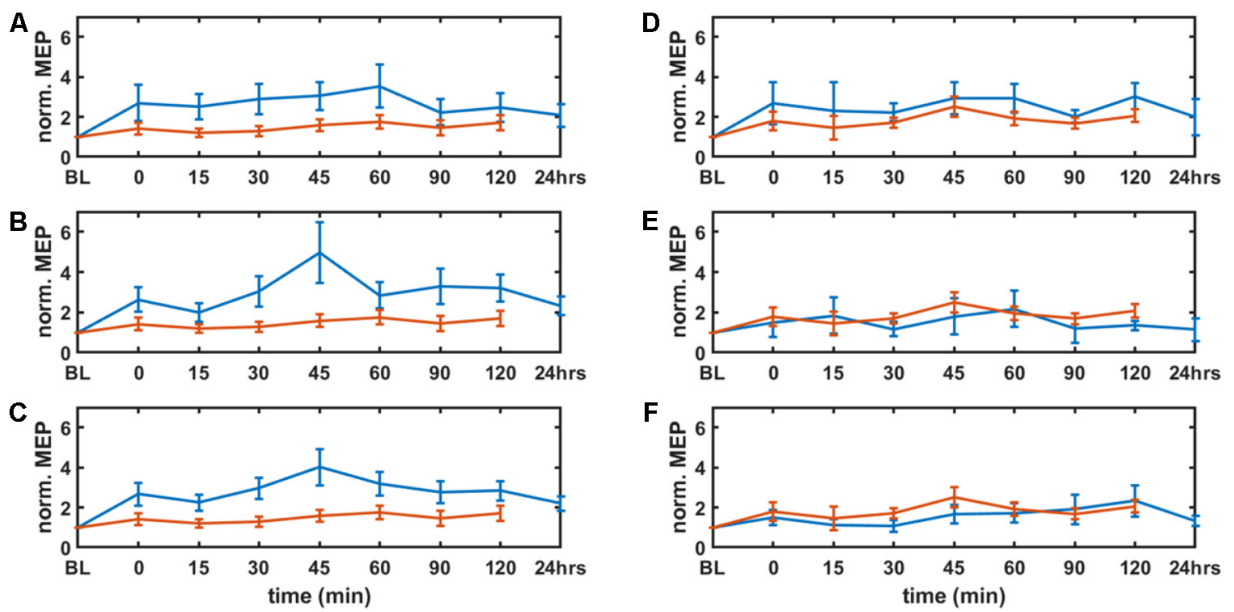

FIGURE 4 | Results from neurophysiological testing - corticospinal excitability alterations evaluated by motor evoked potentials (MEP) from tibialis anterior muscles (left panel: targeted right leg, right panel: contralateral left leg) before and immediately after the completion of tDCS as well as every 15 min for the next 60 min, then every $30 \mathrm{~min}$ for the next $60 \mathrm{~min}$ for each session, and $24 \mathrm{~h}$ for the real stimulation conditions. MEPs were individually normalized to baseline (BL). Solid blue lines show the means of the real tDCS sessions and the solid red lines show the means of the sham tDCS sessions. The error bars show the standard error of means. The parameter space consisted of anode size (large: $5 \mathrm{~cm} \times 7 \mathrm{~cm}$ at $2 \mathrm{~mA}$ and small: $3.5 \mathrm{~cm} \times 1 \mathrm{~cm}$ at $0.2 \mathrm{~mA}$ ), and cathode locations (10-10 EEG system: "side montage" - cathode at T7 and "conventional montage" - cathode at Fp2). A,D show the results of the large-anode "conventional montage," B,E for the small-anode "conventional montage," and C,F show results for the small-anode "side montage."

computational analysis (Figure 3) and neurophysiological testing (Figure 6C). The simulated maximum electric field strength was about one-tenth at the targeted right-leg motor volume with the small-anode (Figures 2B,D), as compared to that induced by the large-anode (Figures 2A,C). Nevertheless, the small-anode montage altered cortical excitability, in agreement 

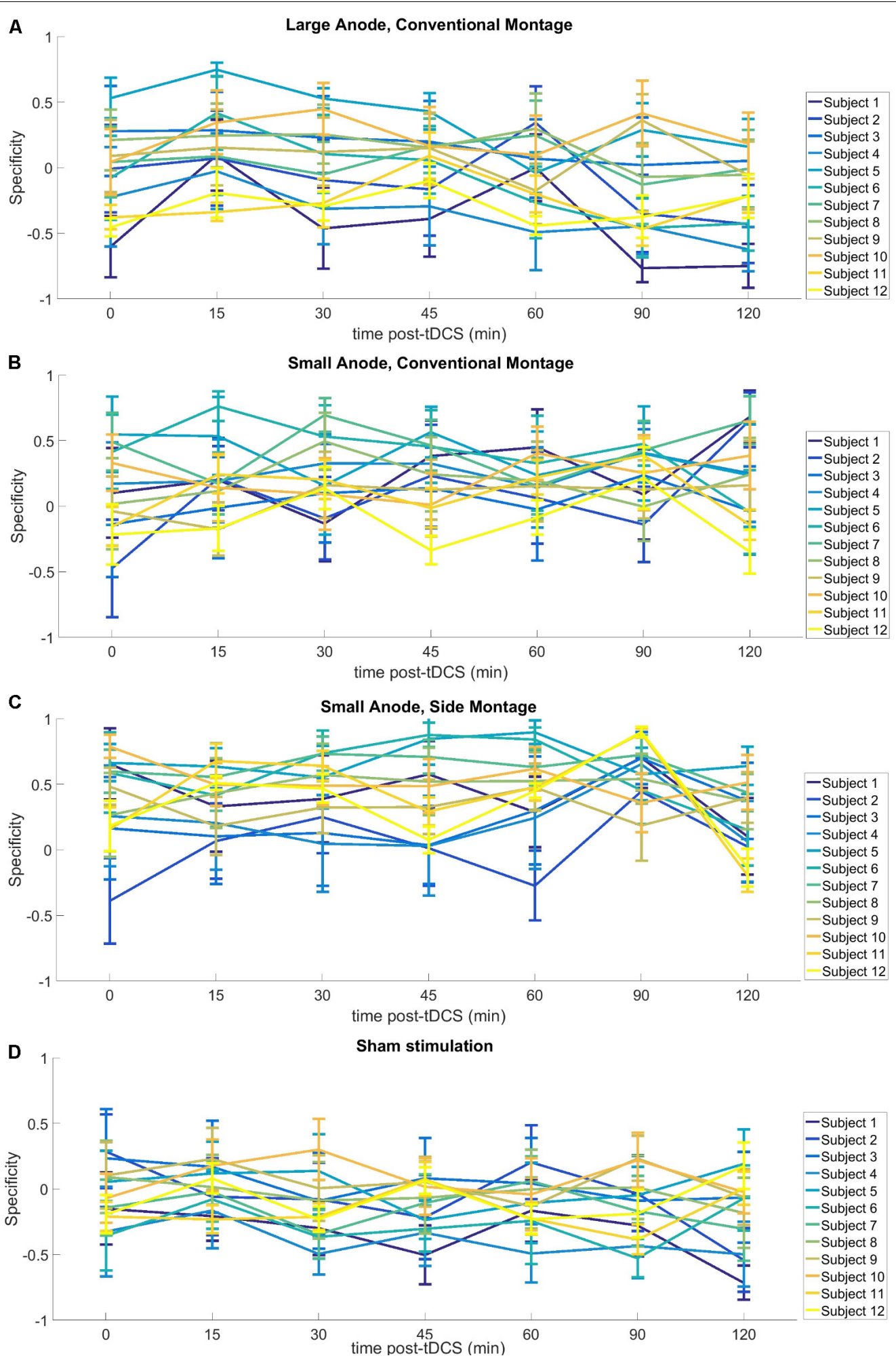

FIGURE 5 | Results from neurophysiological testing - Specificity meurophys - of the corticospinal excitability after-effects for different tDCS-conditions with regard to interindividual variability, (A) large-anode, "conventional montage": large anode, $5 \mathrm{~cm} \times 7 \mathrm{~cm}$ at $2 \mathrm{~mA}$, over the right-leg "target-hotspot" with the cathode over Fp2 (10-10 EEG system), (B) small-anode, "conventional montage": small anode, $3.5 \mathrm{~cm} \times 1 \mathrm{~cm}$ at $0.2 \mathrm{~mA}$, over right-leg "target-hotspot" with the cathode over Fp2 (10-10 EEG system), (C) small anode, "side montage": small-anode, $3.5 \mathrm{~cm} \times 1 \mathrm{~cm}$ at $0.2 \mathrm{~mA}$, over right-leg "target-hotspot" with the cathode over T7 (10-10 EEG system), (D) Sham stimulation: large anode, $5 \mathrm{~cm} \times 7 \mathrm{~cm}$ at $2 \mathrm{~mA}$, at right-leg "target-hotspot" with the cathode at Fp2 (10-10 EEG system). Here, relatively large individual variability is notable, including the sham montage. 

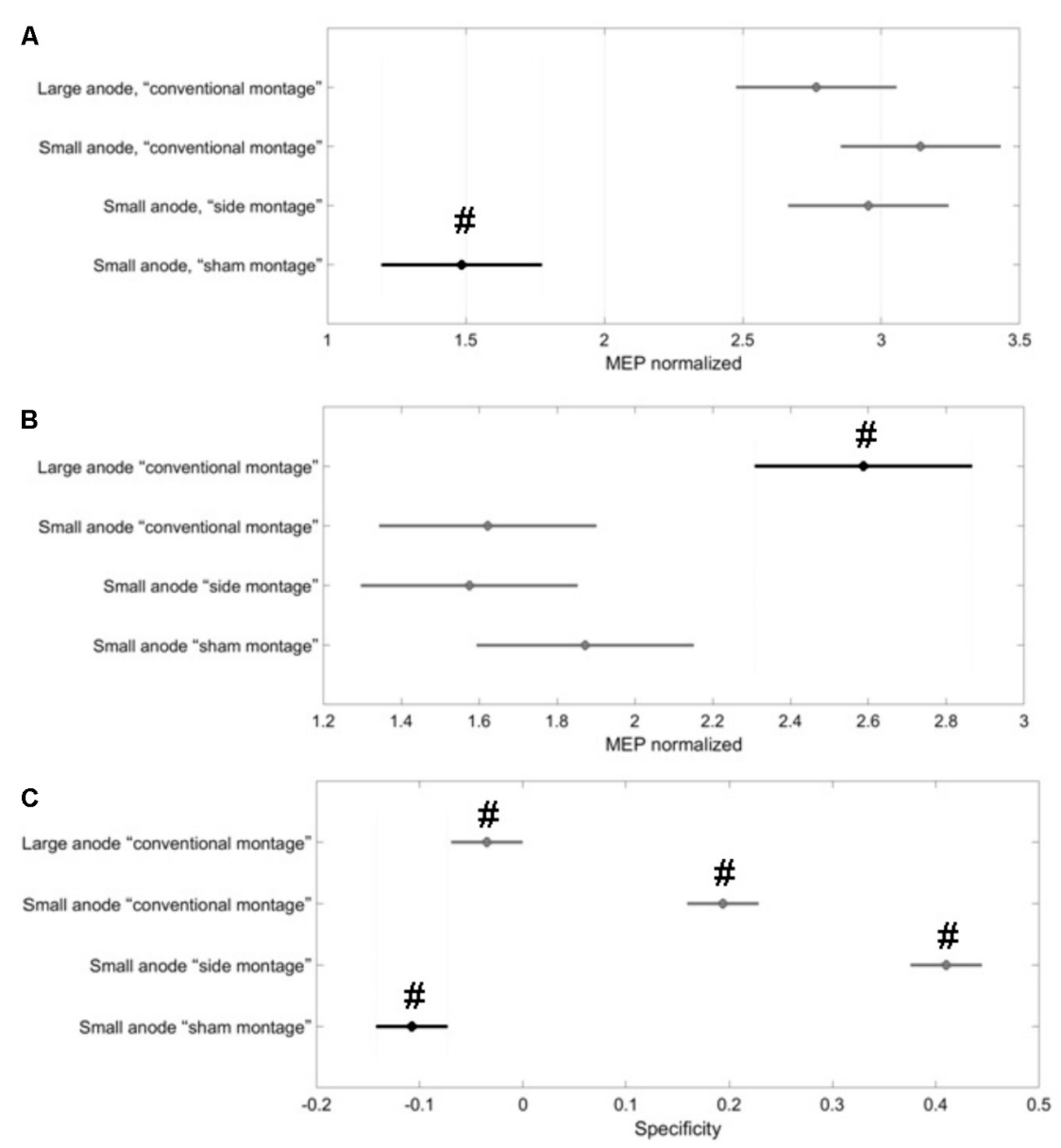

FIGURE 6 | Results from the post hoc tests $(P<0.05)$ presented with 95\% confidence intervals generated from all MEPs. Black represents the data contrasted in the respective post hoc comparison with the other conditions (in gray). Here, two group means are significantly different if their intervals are disjoint; they do not differ significantly if their intervals overlap. (A) The baseline-normalized MEP of the right-leg "target-hotspot" was lowest and significantly different (marked with \#) in the "sham montage" when compared to all real tDCS conditions. (B) the baseline-normalized MEP of the left-leg "contralateral-hotspot" was largest for the large-anode "conventional montage," which differed significantly (marked with \#) from all other tDCS-conditions. (C) Specificity montage each other for all tDCS-conditions (marked with \#), with the small-anode "side montage" having the highest mean, then the small-anode "conventional montage," followed by large-anode "conventional montage" and then, the small-anode "sham montage."

with prior works (Madhavan and Stinear, 2010), in both the "conventional montage" (Figures 4B,E) as well as "side montage" (Figures 4C,F). Therefore, the physiological effects over this target region did not correlate linearly with simulated electrical field (EF) strength. This finding is in accordance with those of a recent study, where it was shown that for a relatively large range of stimulation intensities, anodal tDCS over the motor cortex resulted in similar MEP alterations (Jamil et al., 2017), thus physiological effects may not scale linearly with electric field strength. Alternatively, it cannot be ruled out that the currently available models do not deliver sufficiently correct simulations of EF strength.

In this study, in contrast to the large-anode "conventional montage," the small-anode electrode arrangements resulted in a positive Specificity comp $_{\text {come }}$ in the computational analysis for the "conventional montage" as well as the "side montage," which was confirmed by neurophysiological testing of the Specificity montage $_{\text {comp }}$.
Here, Specificity comp mase defined based on the volumeaveraged magnitude of the electric field or volume-averaged electric field strength (Opitz et al., 2015). Neurophysiological testing confirmed in concurrence with the computational analysis that the small-anode "side montage" provided the best specificity across all evaluated tDCS-conditions: large-anode "conventional montage," small-anode "conventional montage," small-anode "side montage," and small-anode "sham montage." Beyond EF strength that was used to define Specificity comp $_{\text {coge }}$, directionality of the current flow might have relevantly contributed to the specificity differences between electrode arrangements. We found from Figure 3B that tDCS cathode locations over F7, T7, and P7, with the anode over the left primary motor cortex resulted in $\overrightarrow{E F}$ that was primarily in the right-to-left direction in the coronal plane at the right-leg "target-hotspot." This is in accordance with the respective TMS results (Priori et al., 1993) showing that the threshold is lowest for MEPs in the right-leg TA muscle when 
the current in the TMS coil flows from the left to the right side in the coronal plane, i.e., right-to-left oriented induced current in the right-leg "target-hotspot." Figure $\mathbf{3 B}$ shows that the EF direction differs on an average by $11.5^{\circ}$ for the small anode and $9^{\circ}$ for the large anode for the targeted and contralateral ROIs of the "conventional montage" (Fp2 cathode location). Here, the electric field direction is primarily posterior-anterior (PA) (rather than medio-lateral) in the "conventional montage" (Fp2 cathode location). Neurophysiological results showed that MEPs increased comparably in all real tDCS conditions for the targeted leg when compared to sham (see Figure 6A for the targeted leg). However, for the contralateral leg, only the large anode in the conventional montage resulted in a significant increase of MEP as compared to sham stimulation, as shown in Figure 6B. The relatively small difference in EF directions and higher magnitude of the electric field in the large anode conventional montage design, which covers a large volume of the brain including contralateral M1 (caused by the distant anterior position of the return electrode in the "conventional montage") can explain the identically directed effects of stimulation at the targeted and contralateral M1 in this condition. The relatively high magnitude of the EF in this condition $(\sim 0.4 \mathrm{~V} / \mathrm{m}$ with the large anode - see Figure 2) should be sufficient to affect M1 bilaterally. The relatively small difference of EF directions in the right and left motor cortices, most probably caused by the long-distant anterior position of the return electrodes, explains the identically directed effects of stimulation with the large electrodes on both areas, taking also into account that tDCS does not have an effect only on pyramidal neurons, but also on interneurons, which might be directed relevantly in AP/PA directions (Nitsche et al., 2005). The differences between the results of the small and large electrodes with the Fp2 return electrode positions, which resulted in similarly oriented EF vectors, and roughly comparable ipsi- and contralateral EF strength, are most probably caused by different specificity values, as shown by the results of the modeling, where the small electrode resulted in higher specificity in favor of the targeted motor cortex as compared to the large electrode, which resulted in zero specificity. Moreover, the lower absolute EF strength generated by the small electrode according to the modeling results, might have contributed, taking into account that a critical EF strength is assumed to exist, below which no excitability alterations are expected. The specific foundations for these results should, however, be further explored in future studies.

Some limitations of the study should be taken into account. The SimNIBS automated software pipeline (Windhoff et al., 2013) used in this study for computational modeling did not use a subject-specific head model. Therefore, the accuracy of the computed values is limited by the dimensions, the tissues modeled, and the isotropic conductivity values selected for the volume conduction head model. Thus whereas relations between different electrode configurations and placements should be relatively reliable, exact numerical results should be treated with caution. Nevertheless, such simple head models may increase our understanding of how stimulation parameters affect the electric field distribution. For example, Faria et al. (2011) showed that the magnitude of the current density falls more rapidly for smaller electrodes so one will need a higher current density at the electrode to get the same current density (or electric field strength) at deeper cortical targets. In addition, in the "sham montage," we observed an enhancement of post MEP amplitudes, most probably caused by difficulties of the participants to remain completely relaxed regarding muscle tone over the prolonged time course of the experiment. This most likely also resulted in high inter-individual variability in MEP measures (Figure 5). Another factor which might have contributed to this variability is the substantial intrinsic trialto-trial amplitude variability of MEPs, due to state differences of brain activity, and other factors. The recently introduced EEG-adapted stimulation protocols might be helpful to reduce such variability in future (Zrenner et al., 2018). However, the variability of the MEP difference between the targeted and the contralateral RoIs (i.e., the specificity) was not affected as much (as shown in Figure 6C). Nevertheless, the negative specificity in the "sham montage," in Figure 6C, is notable with the nondominant leg showing higher cortical excitability alterations than the dominant leg. This asymmetry might be related to an impact of foot dominance on MEP, similar to results shown for hand dominance in young adults (Bernard and Seidler, 2012). Since only one montage was tested as sham condition, and post-tDCS measures were covering a shorter time course in the sham as in the real stimulation conditions, blinding might potentially have been compromised in some participants; however, the respective multiple-session experimental design and the randomized order of experiments should have prevented unblinding in most participants.

The results of this study might be relevant for presumptive clinical applications of tDCS for reducing post-stroke maladaptive plasticity at the unaffected contralesional hemisphere that produces inter-hemispheric inhibition (Jones, 2017). While, however, higher specificity of stimulation might be achieved relatively easily in non-lesioned brains via modeling of a standard head, and small electrode sizes might be helpful, this does not easily transfer to patients with brain lesions, in which representations of brain functions, and also physical properties of conductivities, might differ. Here, patient-specific individual head-models may be important to optimize tDCS of the leg motor area to make it a viable clinical option in post-stroke neurorehabilitation (Otal et al., 2016).

\section{CONCLUSION}

We conclude that electrode size, cathodal return electrode position have a relevant impact on anodal tDCS effects on excitability of the lower limb motor cortex. In the "conventional montage" condition, the large-anode affected both the targeted and the contralateral leg motor representations in a similar way, while the small-anode in both the "conventional montage" and the "side montage" primarily affected the targeted leg motor representation in terms of corticospinal excitability alterations. Here the "side montage" resulted in more specific effects. The results of this study show that modeling in combination with 
physiological testing is suited to optimize tDCS protocols, and might be relevant for future studies targeting the lower limb motor cortex.

\section{AUTHOR CONTRIBUTIONS}

$\mathrm{MN}$ and $\mathrm{AD}$ contributed to the conception of this investigation. ÁF substantially contributed to the analysis of the electrophysiological data. ZR substantially contributed to the analysis of the computational data. AD, ÁF, WP, and MN

\section{REFERENCES}

Alkadhi, H., Crelier, G. R., Boendermaker, S. H., Golay, X., Hepp-Reymond, M.-C., and Kollias, S. S. (2002). Reproducibility of primary motor cortex somatotopy under controlled conditions. Am. J. Neuroradiol. 23, 1524-1532.

Bailey, N. W., Thomson, R. H., Hoy, K. E., Hernandez-Pavon, J. C., and Fitzgerald, P. B. (2016). TDCS increases cortical excitability: direct evidence from TMS-EEG. Cortex 74, 320-322. doi: 10.1016/j.cortex.2014. 10.022

Batsikadze, G., Moliadze, V., Paulus, W., Kuo, M.-F., and Nitsche, M. A. (2013). Partially non-linear stimulation intensity-dependent effects of direct current stimulation on motor cortex excitability in humans. J. Physiol. 591, 1987-2000. doi: 10.1113/jphysiol.2012.249730

Bernard, J. A., and Seidler, R. D. (2012). Hand dominance and age have interactive effects on motor cortical representations. PLoS One 7:e45443. doi: 10.1371/ journal.pone. 0045443

Bikson, M., Datta, A., Rahman, A., and Scaturro, J. (2010). Electrode montages for tDCS and weak transcranial electrical stimulation: role of "return" electrode's position and size. Clin. Neurophysiol. 121, 1976-1978. doi: 10.1016/j.clinph. 2010.05.020

Collins, D. L., Zijdenbos, A. P., Kollokian, V., Sled, J. G., Kabani, N. J., Holmes, C. J., et al. (1998). Design and construction of a realistic digital brain phantom. IEEE Trans. Med. Imaging 17, 463-468. doi: 10.1109/42.71 2135

Das, S., Holland, P., Frens, M. A., and Donchin, O. (2016). Impact of transcranial direct current stimulation (tDCS) on neuronal functions. Front. Neurosci. 10:550. doi: 10.3389/fnins.2016.00550

Datta, A., Baker, J. M., Bikson, M., and Fridriksson, J. (2011). Individualized model predicts brain current flow during transcranial direct-current stimulation treatment in responsive stroke patient. Brain Stimul. 4, 169-174. doi: 10.1016/j. brs.2010.11.001

Dutta, A., Chugh, S., Banerjee, A., and Dutta, A. (2014a). Point-of-care-testing of standing posture with Wii balance board and microsoft kinect during transcranial direct current stimulation: a feasibility study. NeuroRehabilitation 34, 789-798. doi: 10.3233/NRE-141077

Dutta, A., Paulus, W., and Nitsche, M. A. (2012). "Effects of cathode placement on anodal transcranial DC stimulation of leg motor area: a simulation study," in Proceeding of the 2nd IASTED International Conference Assistive Technology 2012, Innsbruck. doi: 10.2316/P.2012.766-024

Dutta, A., Paulus, W., and Nitsche, M. A. (2014b). Facilitating myoelectric-control with transcranial direct current stimulation: a preliminary study in healthy humans. J. Neuroeng. Rehabil. 11:13. doi: 10.1186/1743-0003-11-13

Fang, Q., and Boas, D. A. (2009). "Tetrahedral mesh generation from volumetric binary and grayscale images," in Proceedings of the IEEE International Symposium on Biomedical Imaging: From Nano to Macro, ISBI'09, Chicago, IL, 1142-1145.

Faria, P., Hallett, M., and Miranda, P. C. (2011). A finite element analysis of the effect of electrode area and inter-electrode distance on the spatial distribution of the current density in tDCS. J. Neural Eng. 8:066017. doi: 10.1088/1741-2560/ $8 / 6 / 066017$

Fischl, B. (2012). FreeSurfer. Neuroimage 62, 774-781. doi: 10.1016/j.neuroimage. 2012.01.021 contributed to the interpretation of the results and writing and reviewing of the manuscript.

\section{FUNDING}

This work was supported by the (i) Alexander von Humboldt Foundation, Germany; (ii) Campus France (PROCOPE Mobility Grants) and INRIA France; and (iii) German Academic Exchange Service (DAAD). The publication of this article was funded by the Open Access Fund of the Leibniz Association.

Flöel, A. (2014). tDCS-enhanced motor and cognitive function in neurological diseases. Neuroimage 85, 934-947. doi: 10.1016/j.neuroimage.2013.05.098

Geuzaine, C., and Remacle, J. F. (2009). Gmsh: a 3-D finite element mesh generator with built-in pre-and post-processing facilities. Int. J. Numer. Methods Eng. 79, 1309-1331. doi: 10.1002/nme.2579

Groppa, S., Oliviero, A., Eisen, A., Quartarone, A., Cohen, L., Mall, V., et al. (2012). A practical guide to diagnostic transcranial magnetic stimulation: report of an IFCN committee. Clin. Neurophysiol. 123, 858-882. doi: 10.1016/j.clinph.2012. 01.010

Holmes, C. J., Hoge, R., Collins, L., Woods, R., Toga, A. W., and Evans, A. C. (1998). Enhancement of MR images using registration for signal averaging. J. Comput. Assist. Tomogr. 22, 324-333. doi: 10.1097/00004728-199803000-00032

Jamil, A., Batsikadze, G., Kuo, H. I., Labruna, L., Hasan, A., Paulus, W., et al. (2017). Systematic evaluation of the impact of stimulation intensity on neuroplastic after-effects induced by transcranial direct current stimulation. J. Physiol. 595, 1273-1288. doi: 10.1113/JP272738

Jayaram, G., and Stinear, J. W. (2009). The effects of transcranial stimulation on paretic lower limb motor excitability during walking. J. Clin. Neurophysiol. 26, 272-279. doi: 10.1097/WNP.0b013e3181af1d41

Jeffery, D. T., Norton, J. A., Roy, F. D., and Gorassini, M. A. (2007). Effects of transcranial direct current stimulation on the excitability of the leg motor cortex. Exp. Brain Res. 182, 281-287. doi: 10.1007/s00221-0071093-y

Jones, T. A. (2017). Motor compensation and its effects on neural reorganization after stroke. Nat. Rev. Neurosci. 18, 267-280. doi: 10.1038/nrn.2017.26

Jurcak, V., Tsuzuki, D., and Dan, I. (2007). 10/20, 10/10, and 10/5 systems revisited: their validity as relative head-surface-based positioning systems. Neuroimage 34, 1600-1611. doi: 10.1016/j.neuroimage.2006.09.024

Kaminski, E., Steele, C. J., Hoff, M., Gundlach, C., Rjosk, V., Sehm, B., et al. (2016). Transcranial direct current stimulation (tDCS) over primary motor cortex leg area promotes dynamic balance task performance. Clin. Neurophysiol. 127, 2455-2462. doi: 10.1016/j.clinph.2016.03.018

Koessler, L., Maillard, L., Benhadid, A., Vignal, J. P., Felblinger, J., Vespignani, H., et al. (2009). Automated cortical projection of EEG sensors: anatomical correlation via the international 10-10 system. Neuroimage 46, 64-72. doi: 10.1016/j.neuroimage.2009.02.006

Liebetanz, D., Nitsche, M. A., Tergau, F., and Paulus, W. (2002). Pharmacological approach to the mechanisms of transcranial DC-stimulation-induced aftereffects of human motor cortex excitability. Brain 125, 2238-2247. doi: 10.1093/ brain/awf 238

Madhavan, S., and Shah, B. (2012). Enhancing motor skill learning with transcranial direct current stimulation-a concise review with applications to stroke. Front. Psychiatry 3:66. doi: 10.3389/fpsyt.2012.00066

Madhavan, S., and Stinear, J. W. (2010). Focal and bidirectional modulation of lower limb motor cortex using anodal transcranial direct current stimulation. Brain Stimul. 3, 42-50. doi: 10.1016/j.brs.2009.06.005

Moliadze, V., Antal, A., and Paulus, W. (2010). Electrode-distance dependent aftereffects of transcranial direct and random noise stimulation with extracephalic reference electrodes. Clin. Neurophysiol. 121, 2165-2171. doi: 10.1016/j.clinph. 2010.04.033

Monte-Silva, K., Kuo, M.-F., Hessenthaler, S., Fresnoza, S., Liebetanz, D., Paulus, W., et al. (2013). Induction of late LTP-like plasticity in the human 
motor cortex by repeated non-invasive brain stimulation. Brain Stimul. 6, 424-432. doi: 10.1016/j.brs.2012.04.011

Monte-Silva, K., Kuo, M.-F., Liebetanz, D., Paulus, W., and Nitsche, M. A. (2010). Shaping the optimal repetition interval for cathodal transcranial direct current stimulation (tDCS). J. Neurophysiol. 103, 1735-1740. doi: 10.1152/jn.00924. 2009

Nitsche, M. A., Fricke, K., Henschke, U., Schlitterlau, A., Liebetanz, D., Lang, N., et al. (2003a). Pharmacological modulation of cortical excitability shifts induced by transcranial direct current stimulation in humans. J. Physiol. 553, 293-301. doi: 10.1113/jphysiol.2003.049916

Nitsche, M. A., Liebetanz, D., Lang, N., Antal, A., Tergau, F., and Paulus, W. (2003b). Safety criteria for transcranial direct current stimulation (tDCS) in humans. Clin. Neurophysiol. 114, 2220-2222. doi: 10.1016/S1388-2457(03) 00235-9

Nitsche, M., and Paulus, W. (2000). Excitability changes induced in the human motor cortex by weak transcranial direct current stimulation. J. Physiol. 527, 633-639. doi: 10.1111/j.1469-7793.2000.t01-1-00633.x

Nitsche, M. A., and Paulus, W. (2001). Sustained excitability elevations induced by transcranial DC motor cortex stimulation in humans. Neurology 57, 1899-1901. doi: 10.1212/WNL.57.10.1899

Nitsche, M. A., Seeber, A., Frommann, K., Klein, C. C., Rochford, C., Nitsche, M. S., et al. (2005). Modulating parameters of excitability during and after transcranial direct current stimulation of the human motor cortex. J. Physiol. 568, 291-303. doi: 10.1113/jphysiol.2005.092429

Oostenveld, R., and Praamstra, P. (2001). The five percent electrode system for high-resolution EEG and ERP measurements. Clin. Neurophysiol. 112, 713-719. doi: 10.1016/S1388-2457(00)00527-7

Opitz, A., Paulus, W., Will, S., Antunes, A., and Thielscher, A. (2015). Determinants of the electric field during transcranial direct current stimulation. Neuroimage 109, 140-150. doi: 10.1016/j.neuroimage.2015.01.033

Otal, B., Dutta, A., Foerster, Á., Ripolles, O., Kuceyeski, A., Miranda, P. C., et al. (2016). Opportunities for guided multichannel non-invasive transcranial current stimulation in poststroke rehabilitation. Front. Neurol. 7:21. doi: 10.3389/fneur.2016.00021

Priori, A., Bertolasi, L., Dressler, D., Rothwell, J., Day, B., Thompson, P., et al. (1993). Transcranial electric and magnetic stimulation of the leg area of the human motor cortex: single motor unit and surface EMG responses in the tibialis anterior muscle. Electroencephalogr. Clin. Neurophysiol. 89, 131-137. doi: 10.1016/0168-5597(93)90095-7

Rawji, V., Ciocca, M., Zacharia, A., Soares, D., Truong, D., Bikson, M., et al. (2018). tDCS changes in motor excitability are specific to orientation of current flow. Brain Stimul. 11, 289-298. doi: 10.1016/j.brs.2017.11.001

Roche, N., Lackmy, A., Achache, V., Bussel, B., and Katz, R. (2011). Effects of anodal transcranial direct current stimulation over the leg motor area on lumbar spinal network excitability in healthy subjects. J. Physiol. 589, 2813-2826. doi: 10.1113/jphysiol.2011.205161

Rossi, S., Hallett, M., Rossini, P. M., Pascual-Leone, A., and Safety of TMS Consensus Group (2009). Safety, ethical considerations, and application guidelines for the use of transcranial magnetic stimulation in clinical practice and research. Clin. Neurophysiol. 120, 2008-2039. doi: 10.1016/j.clinph.2009. 08.016

Rossini, P., Berardelli, A., Deuschl, G., Hallett, M., Maertens de Noordhout, A., Paulus, W., et al. (1999). Applications of magnetic cortical stimulation. Electroencephalogr. Clin. Neurophysiol. Suppl. 52, 171-185.

Sohn, M. K., Jee, S. J., and Kim, Y. W. (2013). Effect of transcranial direct current stimulation on postural stability and lower extremity strength in hemiplegic stroke patients. Ann. Rehabil. Med. 37, 759-765. doi: 10.5535/arm.2013.37. 6.759

Tanaka, S., Hanakawa, T., Honda, M., and Watanabe, K. (2009). Enhancement of pinch force in the lower leg by anodal transcranial direct current stimulation. Exp. Brain Res. 196, 459-465. doi: 10.1007/s00221-009-1863-9

Tanaka, S., Takeda, K., Otaka, Y., Kita, K., Osu, R., Honda, M., et al. (2011). Single session of transcranial direct current stimulation transiently increases knee extensor force in patients with hemiparetic stroke. Neurorehabil. Neural Repair 25, 565-569. doi: 10.1177/1545968311402091

van Asseldonk, E. H., and Boonstra, T. A. (2016). Transcranial direct current stimulation of the leg motor cortex enhances coordinated motor output during walking with a large inter-individual variability. Brain Stimul. 9, 182-190. doi: 10.1016/j.brs.2015.10.001

Windhoff, M., Opitz, A., and Thielscher, A. (2013). Electric field calculations in brain stimulation based on finite elements: an optimized processing pipeline for the generation and usage of accurate individual head models. Hum. Brain Mapp. 34, 923-935. doi: 10.1002/hbm.21479

Zrenner, C., Desideri, D., Belardinelli, P., and Ziemann, U. (2018). Real-time EEG-defined excitability states determine efficacy of TMS-induced plasticity in human motor cortex. Brain Stimul. 11, 374-389. doi: 10.1016/j.brs.2017.11.016

Conflict of Interest Statement: The authors declare that the research was conducted in the absence of any commercial or financial relationships that could be construed as a potential conflict of interest.

Copyright (c) 2018 Foerster, Rezaee, Paulus, Nitsche and Dutta. This is an open-access article distributed under the terms of the Creative Commons Attribution License (CC BY). The use, distribution or reproduction in other forums is permitted, provided the original author(s) and the copyright owner(s) are credited and that the original publication in this journal is cited, in accordance with accepted academic practice. No use, distribution or reproduction is permitted which does not comply with these terms. 